\title{
Parameter-Independent Dynamical Behaviors in Memristor-Based Wien-Bridge Oscillator
}

\author{
Ning Wang, Bocheng Bao, Tao Jiang, Mo Chen, and Quan Xu \\ School of Information Science and Engineering, Changzhou University, Changzhou 213164, China \\ Correspondence should be addressed to Bocheng Bao; mervinbao@126.com
}

Received 9 August 2017; Accepted 9 December 2017; Published 26 December 2017

Academic Editor: Alessandro Lo Schiavo

Copyright (C) 2017 Ning Wang et al. This is an open access article distributed under the Creative Commons Attribution License, which permits unrestricted use, distribution, and reproduction in any medium, provided the original work is properly cited.

\begin{abstract}
This paper presents a novel memristor-based Wien-bridge oscillator and investigates its parameter-independent dynamical behaviors. The newly proposed memristive chaotic oscillator is constructed by linearly coupling a nonlinear active filter composed of memristor and capacitor to a Wien-bridge oscillator. For a set of circuit parameters, phase portraits of a double-scroll chaotic attractor are obtained by numerical simulations and then validated by hardware experiments. With a dimensionless system model and the determined system parameters, the initial condition-dependent dynamical behaviors are explored through bifurcation diagrams, Lyapunov exponents, and phase portraits, upon which the coexisting infinitely many attractors and transient chaos related to initial conditions are perfectly offered. These results are well verified by PSIM circuit simulations.
\end{abstract}

\section{Introduction}

As the fourth basic circuit element, memristor, describing the relationship between the electric charge and magnetic flux, had been predicted by Chua in 1971 [1]. The remarkable characteristic is that memristor is a new two-terminal element with variable resistance depending on the amount of electric charge passing through it in a determinate direction. Thus, memristors are conveniently led into some nonlinear electronic circuits to replace nonlinear elements to design novel chaotic circuits. In recent years, novel nonlinear dynamical behaviors, like chaos and hyperchaos [2-7], transient chaos $[8]$, self-excited and hidden attractor $[9,10]$, coexisting multiple attractors $[11,12]$, and so on, have been reported in several memristor-based circuits.

Particularly, multistability, that is, coexisting multiple attractors, is an interesting phenomenon appearing in various physical fields, for example, power system [11], nonlinear system/circuit [12-22], and neural networks [23-25], which means that there are more than two different types of coexisting attractors. Sequentially, the phenomenon called extreme multistability occurs when the number of coexisting attractors tends to be infinite for the same set of circuit/system parameters [26-34]. Due to the important and potential values of engineering applications [35-37], study on revealing the phenomenon of coexisting attractors and investigating the internal mechanism of extreme multistability has attracted great attention and become a research focus [2637]. Furthermore, transient chaos is a common phenomenon in many nonlinear dynamical systems $[8,30,38-43]$. It is necessary to study the transient dynamics to avoid some unwanted thing happening in a finite period of time before settling into final state, for example, the situations of voltage collapse [43]. At the same time, it will be useful to generate more flexible random signals for secret communications and information encryptions.

Chua's circuit, containing a nonlinear element called Chua's diode, is the first electronic circuit for generating double-scroll chaotic attractor [44]. By replacing the Chua's diode with a memristor, some memristor-based chaotic circuits have been reported [8-10, 20, 23, 32-34]. The novel characteristic of memristor-based chaotic circuit is that its dynamical behavior is closely related to the initial state of memristor [19, 20, 29-34]. That is to say, with the variation of memristor initial condition, various kinds of attractors may coexist under the determined circuit/system parameters. Based on the above considerations, a novel memristive chaotic circuit is constructed in this paper. Naturally, complex parameter-independent dynamics, for example, coexisting 


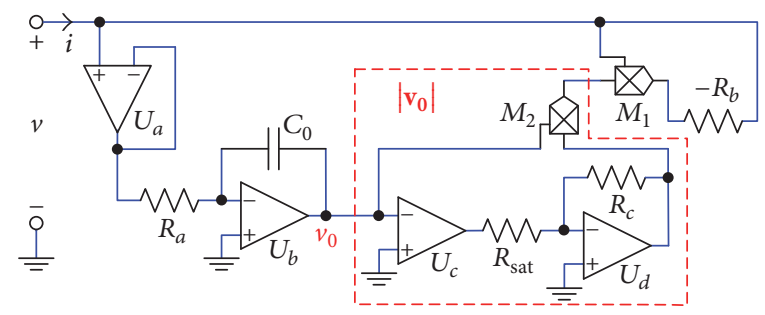

FIGURE 1: Equivalent circuit of active voltage-controlled memristor.

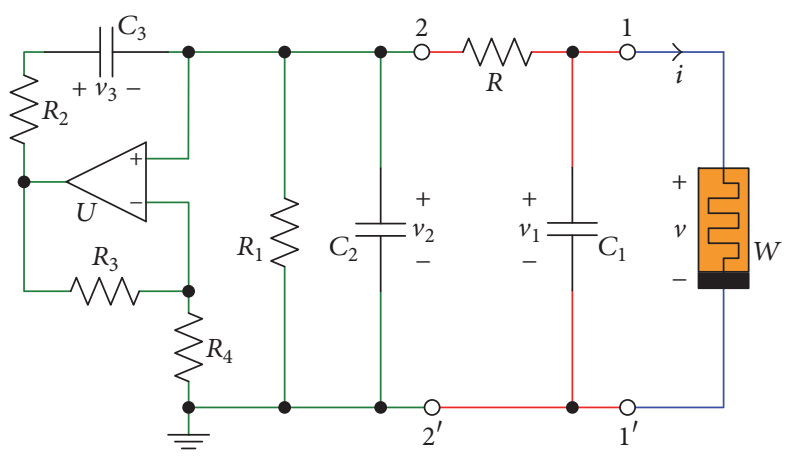

FIgURE 2: Memristor-based Wien-bridge oscillator.

infinitely many attractors' behavior and transient transition behavior, can be found.

The layout of the paper is as follows. Section 2 proposes a memristor-based chaotic circuit with line equilibrium and gives the circuit state equations. Numerical simulations and hardware experiments are utilized to observe its strange attractors. In Section 3, a dimensionless model is carried out via introducing four new variables and rescaling the circuit parameters to perform stability analysis of the line equilibrium. In Section 4, coexisting infinitely many attractors' behavior and transient transition behavior are exhibited through parameter-independent bifurcation diagrams, phase portraits, and time-domain waveform. PSIM circuit simulations are utilized to verify the phenomenon of extreme multistability. Section 5 gives some concluded remarks and outlooks.

\section{Memristor-Based Wien-Bridge Oscillator}

2.1. Circuit Descriptions and State Equations. An active voltage-controlled memristor reported in [2] is utilized in this paper. The equivalent circuit of the memristor is shown in Figure 1, where the circuit in the dotted box is used to implement the absolute value function and its circuit parameters are selected as $R_{c}=10 \mathrm{k} \Omega, R_{\text {sat }}=13.5 \mathrm{k} \Omega$ and the gain of the analogue multiplier $M_{2}$ is assigned as $g_{2}=0.1$. Note that the operational amplifier saturation voltage is $E_{\text {sat }}$ $= \pm 13.5 \mathrm{~V}$ for $\pm 15 \mathrm{~V} \mathrm{DC}$ power supplies.

Let $v$ and $i$ be the voltage and the current at the input port, respectively, and $v_{0}$ stand for the voltage across the capacitor $C_{0}$. The voltage-current relationship is thereby characterized as

$$
i=W\left(v_{0}\right) v=-\frac{1}{R_{b}}\left(1-g\left|v_{0}\right|\right) v
$$

$$
C_{0} \frac{\mathrm{d} v_{0}}{\mathrm{~d} t}=-\frac{1}{R_{a}} v
$$

where $g$ is the gain of the analogue multiplier $M_{1}$.

By replacing simplified Chua's diode reported in [45] with the memristor depicted in Figure 1, a memristor-based Wienbridge oscillator is derived, as shown in Figure 2. The right part of the port $11^{\prime}$ is the active voltage-controlled memristor, and the left part of the port $22^{\prime}$ is the classical Wien-bridge oscillator.

The state equations for the proposed circuit can be written by four first-order differential equations as

$$
\begin{aligned}
& C_{1} \frac{\mathrm{d} v_{1}}{\mathrm{~d} t}=-\frac{1}{R} v_{1}+\frac{1}{R} v_{2}+\frac{1}{R_{b}}\left(1-g\left|v_{0}\right|\right) v_{1}, \\
& C_{2} \frac{\mathrm{d} v_{2}}{\mathrm{~d} t}=\frac{1}{R} v_{1}+\left(\frac{k}{R_{2}}-\frac{1}{R^{\prime}}\right) v_{2}-\frac{1}{R_{2}} v_{3}, \\
& C_{3} \frac{\mathrm{d} v_{3}}{\mathrm{~d} t}=\frac{k}{R_{2}} v_{2}-\frac{1}{R_{2}} v_{3}, \\
& C_{0} \frac{\mathrm{d} v_{0}}{\mathrm{~d} t}=-\frac{1}{R_{a}} v_{1},
\end{aligned}
$$

where, $v_{0}, v_{1}, v_{2}$, and $v_{3}$ are four state variables representing the voltages of capacitors $C_{0}, C_{1}, C_{2}$, and $C_{3}$, respectively, $k=$ $R_{3} / R_{4}$, and $R^{\prime}=R R_{1} /\left(R+R_{1}\right)$.

The typical circuit parameters in Figures 1 and 2 are listed in Table 1.

2.2. Numerical Simulations and Hardware Experiments. In this part, Runge-Kutta ODE23 algorithm is utilized to draw 
TABLE 1: Circuit parameters of the memristor-based Wien-bridge oscillator.

\begin{tabular}{lcc}
\hline Parameters & Significations & Values \\
\hline$C_{0}, C_{1}$ & Capacitance & $6.8 \mathrm{nF}$ \\
$C_{2}, C_{3}$ & Capacitance & $100 \mathrm{nF}$ \\
$R$ & Resistance & $2 \mathrm{k} \Omega$ \\
$R_{1}, R_{2}$ & Resistance & $400 \Omega$ \\
$R_{3}$ & Resistance & $6.6 \mathrm{k} \Omega$ \\
$R_{4}$ & Resistance & $3 \mathrm{k} \Omega$ \\
$R_{a}$ & Resistance & $5 \mathrm{k} \Omega$ \\
$R_{b}$ & Resistance & $1.6 \mathrm{k} \Omega$ \\
$g$ & Scale factor of $M_{1}$ & 0.1 \\
\hline
\end{tabular}

phase portraits. The circuit parameters listed in Table 1 are used for numerical simulations and hardware experiments. The initial conditions of four state variables are set to $\left(10^{-9} \mathrm{~V}\right.$, $0 \mathrm{~V}, 0 \mathrm{~V}, 0 \mathrm{~V})$. The four Lyapunov exponents are calculated by Wolf et al.s method [46] as $L_{1}=2.0759 \times 10^{3}, L_{2}=$ 11.7050, $L_{3}=-8.6321$, and $L_{4}=-3.2043 \times 10^{4}$, indicating that chaos emerges in the proposed memristor-based Wienbridge oscillator. A double-scroll chaotic attractor can be numerically simulated and plotted in different phase planes, as shown in Figure 3.

According to the equivalent circuit of the memristor in Figure 1 and the circuit schematic of the memristor-based Wien-bridge oscillator in Figure 2, a common hardware circuit on breadboards is designed to validate the dynamical behaviors. Figure 4 is a photograph of the experimental hardware circuit and a double-scroll chaotic attractor captured by the digital oscilloscope. In this circuit, operational amplifier AD711KN and analogue multiplier AD633JN are selected and supplied with $\pm 15 \mathrm{~V}$ DC power supplies. The experimental results are captured by four-channel digital oscilloscope. The circuit parameters listed in Table 1 are utilized; experimentally measured phase portraits of the typical chaotic attractor are shown in Figure 5, which are well consistent with numerical simulations plotted in Figure 3.

\section{Dimensionless System Model}

To preferably investigate parameter-independent dynamical behaviors in memristor-based Wien-bridge oscillator, a dimensionless system model is necessary, by introducing four new variables and rescaling the circuit parameters in a dimensionless form as

$$
\begin{aligned}
x & =v_{1}, \\
y & =v_{2}, \\
z & =v_{3}, \\
w & =\frac{R_{a} v_{0}}{R}, \\
\dot{u} & =\frac{\mathrm{d} u}{\mathrm{~d} \tau} \quad(u \equiv x, y, z, w),
\end{aligned}
$$

$$
\begin{aligned}
C_{2} & =C_{3}=C, \\
\tau & =\frac{t}{(R C)}, \\
p & =\frac{R}{R_{b}}, \\
q & =\frac{g R^{2}}{\left(R_{a} R_{b}\right)}, \\
a & =\frac{C}{C_{0}}=\frac{C}{C_{1}}, \\
b & =\frac{R}{R_{1}}=\frac{R}{R_{2}}, \\
k & =\frac{R_{3}}{R_{4}} .
\end{aligned}
$$

Equation (2) can be rewritten as

$$
\begin{aligned}
\dot{x} & =-a(x-y)-a W(w) x, \\
\dot{y} & =x+[(k-1) b-1] y-b z, \\
\dot{z} & =b(k y-z), \\
\dot{w} & =-a x,
\end{aligned}
$$

where $W(w)=-p+q|w|$. Therefore, the normalized parameters for (4) are obtained by (3) as

$$
\begin{aligned}
& a=\frac{250}{17}, \\
& b=5, \\
& p=1.25, \\
& q=0.05, \\
& k=2.2 .
\end{aligned}
$$

By solving the equations $\dot{x}=\dot{y}=\dot{z}=\dot{w}=0$, we can obtain the equilibrium of system (4). Clearly, the system has a line equilibrium, which is expressed as

$$
P=\{(x, y, z, w) \mid x=y=z=0, w=\mu\},
$$

where $\mu$ is an uncertain constant. For the line equilibrium $P$, the Jacobian matrix is given as

$$
\mathbf{J}=\left[\begin{array}{cccc}
-a-a(-p+q|\mu|) & a & 0 & 0 \\
1 & (k-1) b-1 & -b & 0 \\
0 & k b & -b & 0 \\
-a & 0 & 0 & 0
\end{array}\right] .
$$

Correspondingly, the characteristic equation used to solve four eigenvalues is obtained as

$$
\operatorname{det}(\mathbf{1} \lambda-\mathbf{J})=\lambda\left(\lambda^{3}+\varepsilon_{1} \lambda^{2}+\varepsilon_{2} \lambda+\varepsilon_{3}\right)=0,
$$




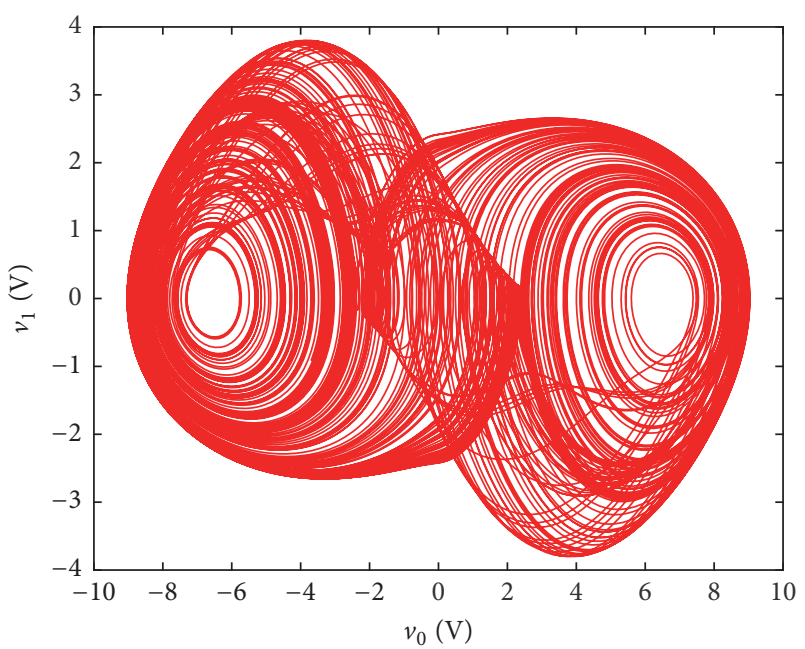

(a)

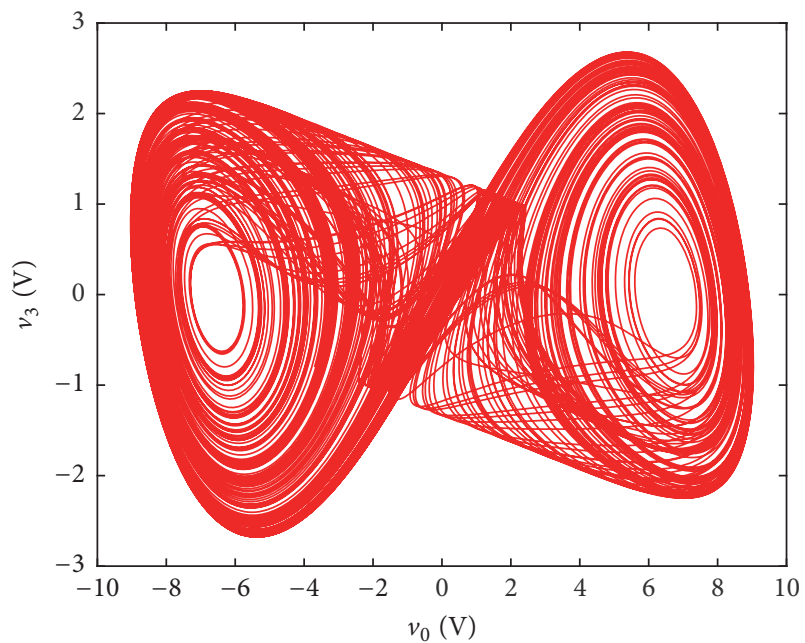

(c)

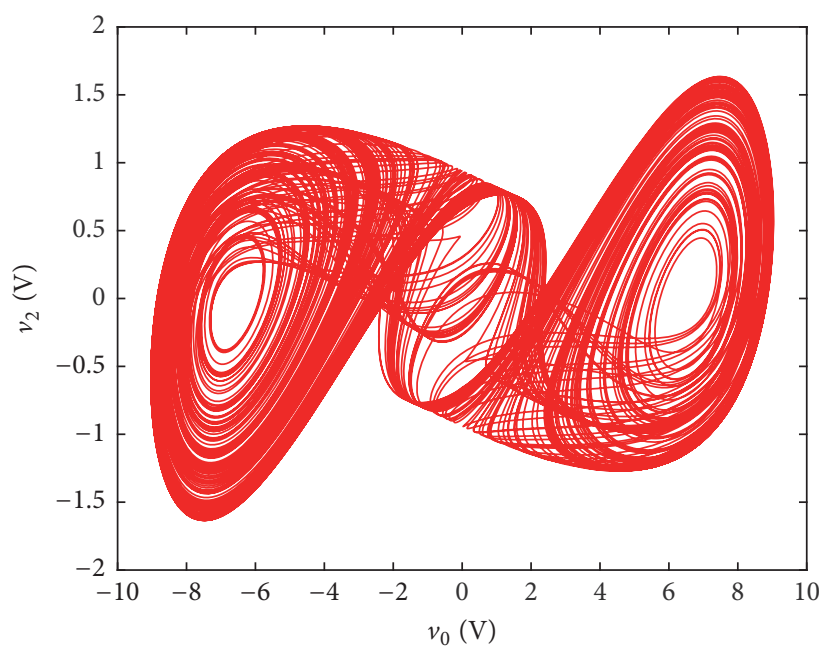

(b)

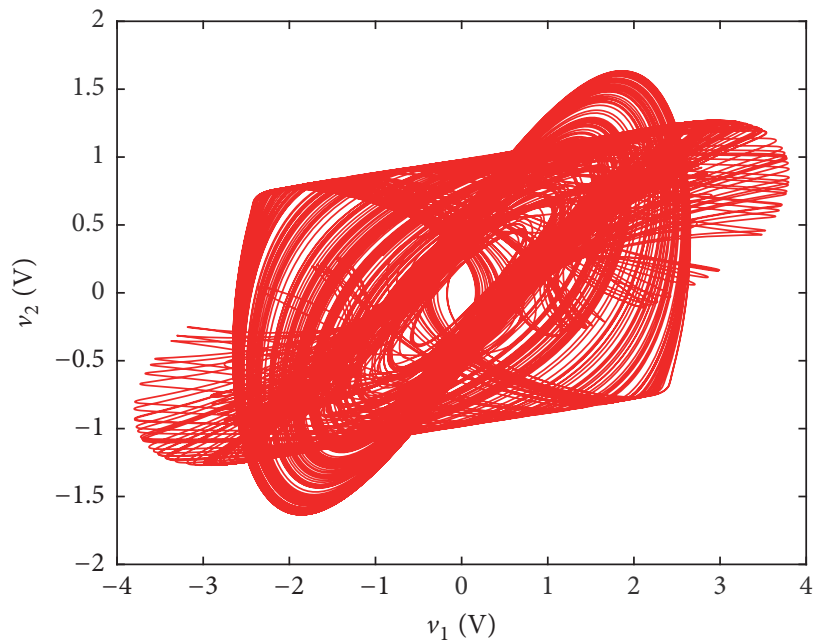

(d)

FIgURE 3: Numerical phase portraits of chaotic attractor in different planes, where the circuit parameters are given in Table 1 . (a) The $v_{0}-v_{1}$ plane; (b) the $v_{0}-v_{2}$ plane; (c) the $v_{0}-v_{3}$ plane; (d) the $v_{1}-v_{2}$ plane.

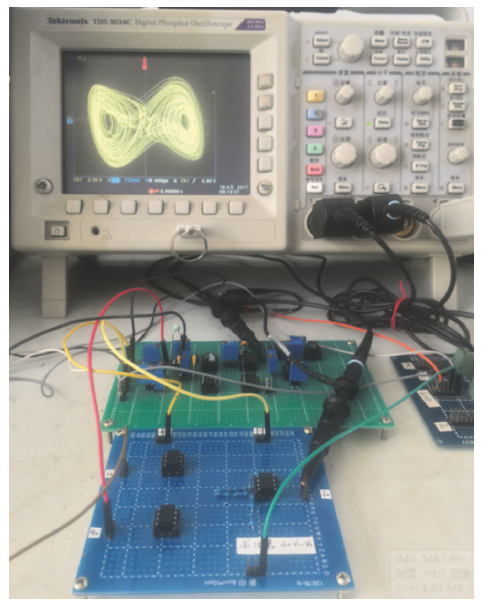

FIGURE 4: Photograph for experimental hardware circuit connecting digital oscilloscope, where the green board is main circuit and the blue board is a subtracter for measuring $v_{3}$. 


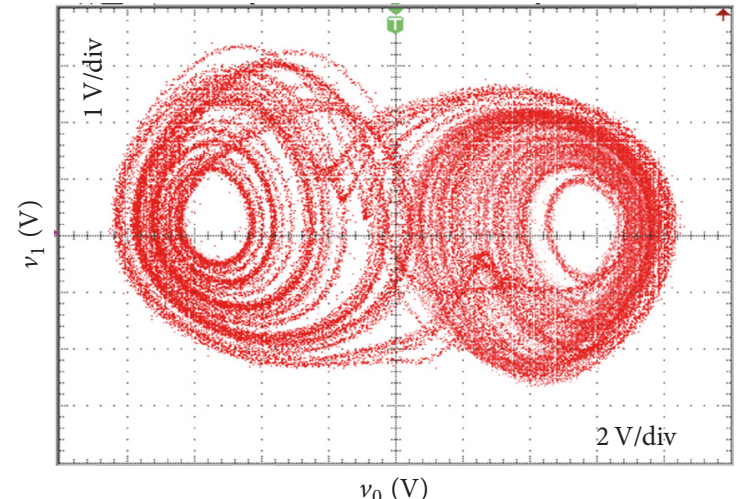

(a)

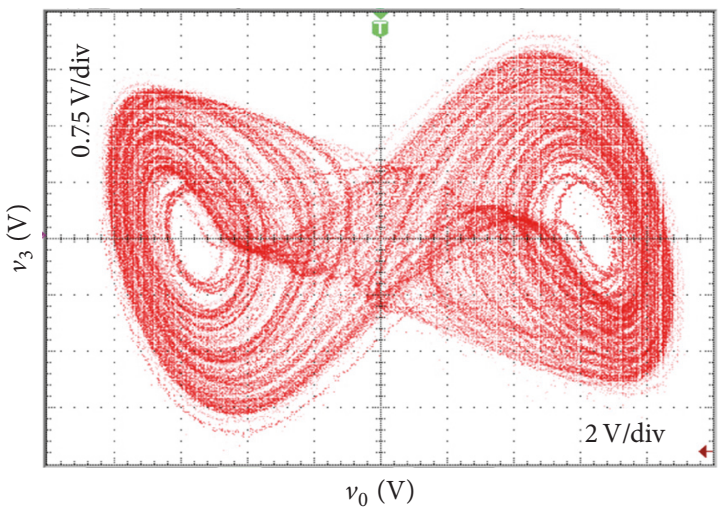

(c)

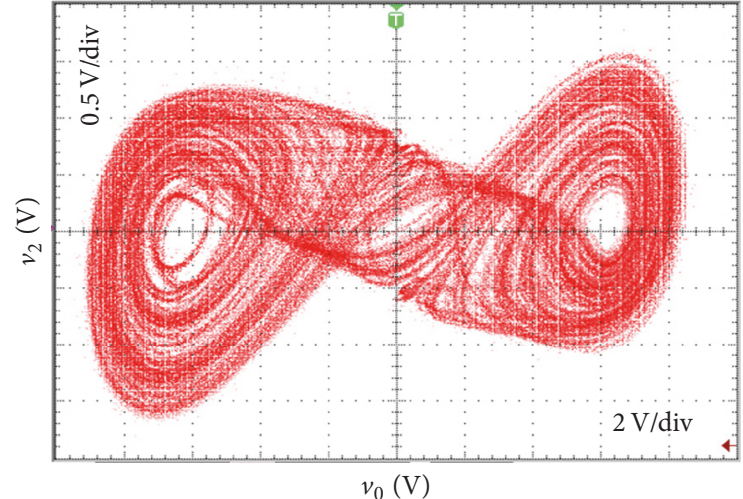

(b)

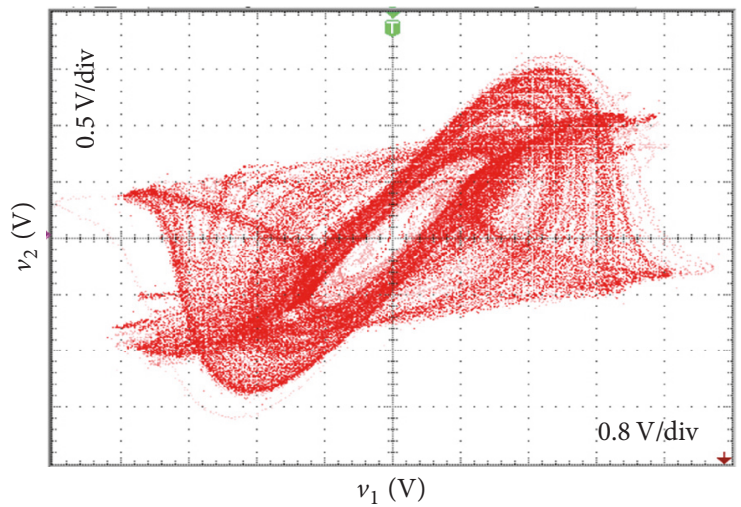

(d)

FiguRE 5: Experimental phase portraits of the chaotic attractor in different planes, where the circuit parameters are given by Table 1. (a) The $v_{0}-v_{1}$ plane; (b) the $v_{0}-v_{2}$ plane; (c) the $v_{0}-v_{3}$ plane; (d) the $v_{1}-v_{2}$ plane.

where

$$
\begin{aligned}
& \varepsilon_{1}=a+a \eta-k b+2 b+1, \\
& \varepsilon_{2}=b(b+1-k a+2 a)-\eta a(k b-2 b-1), \\
& \varepsilon_{3}=a b(\eta b+\eta+b), \\
& \eta=-p+q|\mu| .
\end{aligned}
$$

There are one zero root and three nonzero roots in characteristic equation (8). For these nonzero roots, the stability conditions via Routh-Hurwitz criterion are given as

$$
\begin{aligned}
\varepsilon_{1} & >0, \\
\varepsilon_{3} & >0, \\
\varepsilon_{1} \varepsilon_{2}-\varepsilon_{3} & >0 .
\end{aligned}
$$

If the three conditions of (10) are satisfied, the line equilibrium $P$ is stable, whereas if any one of the three conditions is not satisfied, $P$ is unstable, which may induce the emergence of periodic and chaotic behaviors in system (4). When $\mu$ varies in the region $(-25 / 3,25 / 3)$ system (4) is unstable, and the corresponding four eigenvalues at equilibrium $P$ have a positive real root, two complex conjugate roots with negative real parts, and a zero root.

\section{Parameter-Independent Bifurcation Behaviors}

In this section, bifurcation behaviors associated with the initial conditions are investigated by numerical simulations. The normalized parameters listed in (5) are fixed, and the state variable $x$ is chosen as bifurcation variable. Runge-Kutta ODE23 algorithm is used for numerical calculations and Wolf et al.s method [46] is used to calculate Lyapunov exponents.

4.1. Memristor Initial Condition-Dependent Dynamics. In order to study the dynamical behavior of the memristorbased Wien-bridge oscillator under different memristor initial condition, three initial conditions $x(0)=0, y(0)=0$, and $z(0)=10^{-9}$ are fixed, and the initial condition $w(0)$ is taken to vary in the region $[-8,8]$. With $w(0)$ increasing, the bifurcation diagram and the four Lyapunov exponents are plotted in Figures 6(a) and 6(b), respectively.

From Figure 6, complex bifurcation behaviors including tangent bifurcation, period doubling bifurcation, reverse period doubling bifurcation, and chaotic crisis can be observed, and the coexistence of point attractors with different locations, periodic limit cycles with different periodicities, and chaotic attractors with different topologies can be found. When $w(0)$ is increased continuously, system (4) starts 


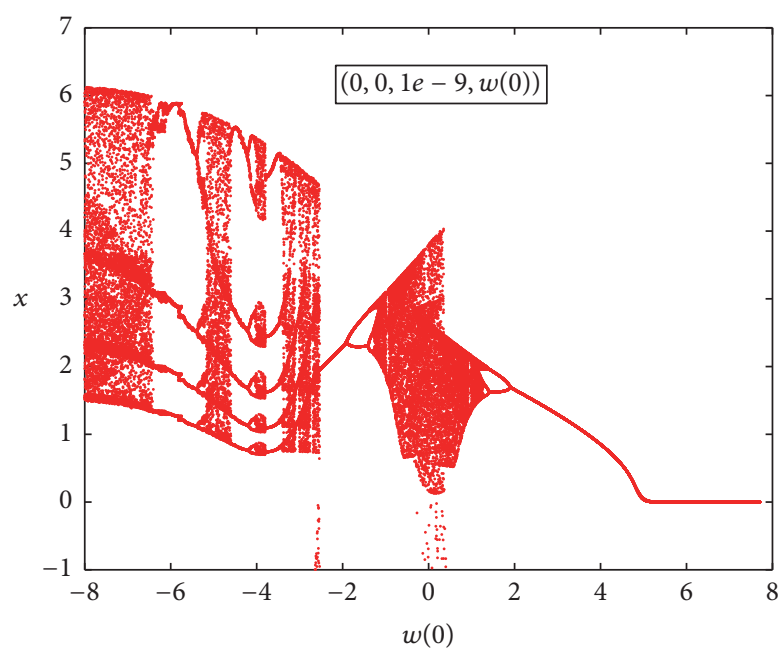

(a)

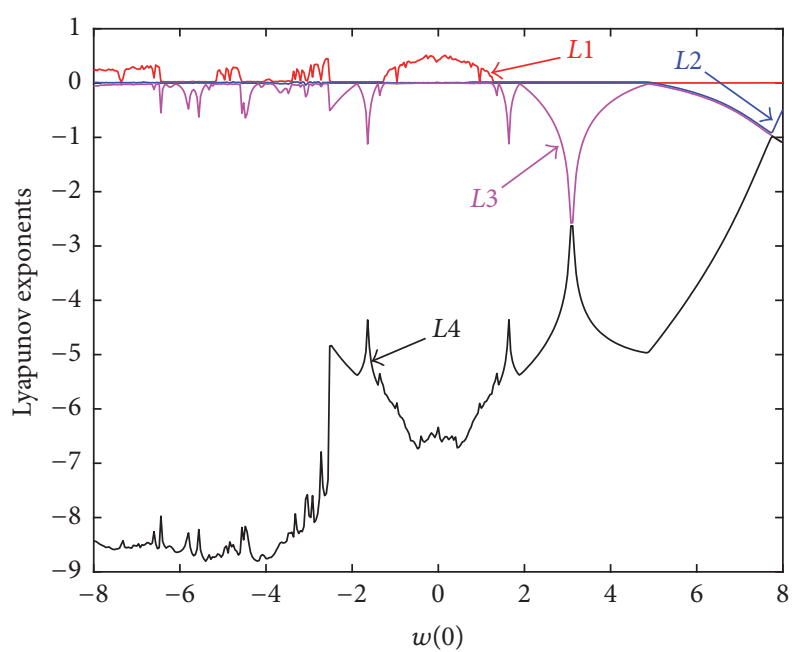

(b)

FIGURE 6: Dynamics with the variation of memristor initial condition $w(0)$. (a) Bifurcation diagram; (b) Lyapunov exponent spectra.

from the chaotic state and breaks into period 4 state through tangent bifurcation at $w(0)=-6.54$ and then goes into chaotic state via period doubling bifurcation. Afterwards, at $w(0)=-4.66$, system (4) breaks into period 5 state via the second tangent bifurcation and then goes through period doubling bifurcation and reverse period doubling bifurcation with the emergence of five bubbles in the initial region of $[-4.66,-3.46]$ [31]. Furthermore, a chaotic crisis occurs at $w(0)=-2.58$, which results in the fact that system (4) jumps from chaotic state into periodic state. It is interesting that, within the initial region of $[-2.58,2.58]$, system (4) goes through period doubling bifurcation to reverse period doubling bifurcation and exhibits symmetrical dynamics about $w(0)=0$ which leads to the coexistence of symmetrical attractors. Finally, system (4) tends to the stable state with point attractor at $w(0)=5.07$.

To confirm the coexisting attractors, phase portraits under different memristor initial conditions are plotted, as shown in Figure 7. Figures 7(a) and 7(b) show the route to chaos, for example, period doubling bifurcation route and reverse period doubling bifurcation route. Both the two routes are symmetrical in the regions $[-2.58,0]$ and $[0,2.58]$. It can be observed from Figure 7 (a) that when $w(0)$ is set to $2.4,1.4$, and 0.5 and $-2.4,-1.4$, and -0.5 , respectively, the corresponding attractors in the $w-x$ plane are origin-symmetric. Figure $7(b)$ is a double-scroll chaotic attractor under the memristor initial condition $w(0)=0.001$. Furthermore, Figures $7(\mathrm{c})$ and $7(\mathrm{~d})$ are plotted to show other coexisting attractors in the regions of $[-8,-2.58)$ and $(2.58,8]$.

It is obvious that the many infinitely coexisting attractors' behavior can be found via memristor initial conditionbased bifurcation dynamics in Figure 6 and confirmed by phase portraits in Figure 7. Considering that the attractors plotted in Figure 7 are shaped under the partly given system initial conditions, practically, there are infinitely many coexisting attractors when the initial condition $w(0)$ changes continuously. The coexisting phenomenon of infinitely many attractors is also known as extreme multistability [26-31].

Comparing Figure 6(a) with Figure 6(b), we find that when the initial condition $w(0)=-7.35$, bifurcation dynamics in Figure 6(a) shows the chaotic behavior, but the largest Lyapunov exponent $L_{1}$ in Figure 6(b) drops to zero and indicates the periodic behavior. Different dynamical behaviors imply that there is a transition from transient chaotic to steady periodic behaviors with the evolution of the time.

To reveal this interesting transition behavior, the timedomain waveform and phase portraits are plotted in Figure 8. Figure 8(a) shows the time-domain waveform of the variable $x$ in the time interval $[0 \mathrm{ks}, 2 \mathrm{ks}]$, from which it can be obviously observed that the system trajectory starts from a chaotic orbit and then enters into a periodic orbit at $1.33 \mathrm{ks}$. Figures $8(b)$ and $8(c)$ show the phase portraits in two different time intervals of [0.6 ks, $1 \mathrm{ks}]$ and $[1.6 \mathrm{ks}, 2 \mathrm{ks}]$, respectively.

\subsection{Other Initial Conditions-Relevant Dynamical Behaviors.} Three initial values are chosen as $y(0)=0, z(0)=10^{-9}$, and $w(0)=0$. When the initial condition $x(0)$ is gradually adjusted from -2 to 2 , the bifurcation diagram and the corresponding four Lyapunov exponent spectra are shown in Figures 9(a) and $9(\mathrm{~b})$, respectively.

While increasing the initial condition $x(0)$ from -2 to 2 , such dynamical behaviors as period, chaos, period doubling bifurcation, reverse period doubling bifurcation, and tangential bifurcation can be observed from Figure 9(a). In the region $[-0.9,0.9]$, period doubling bifurcation and reverse period doubling bifurcation can be observed clearly, leading to the occurrence of symmetrical coexisting attractors, as plotted in Figure 10, where Figures 10(a)-10(d) show the dynamics from period to chaos through period doubling bifurcation, and Figures 10(e)-10(h) display limit cycles with different periodicities and chaotic spiral attractors. Note that phase portraits with the red and blue orbits represent their initial conditions as $\left(-x(0), 0,10^{-9}, 0\right)$ and $\left(x(0), 0,10^{-9}, 0\right)$, 


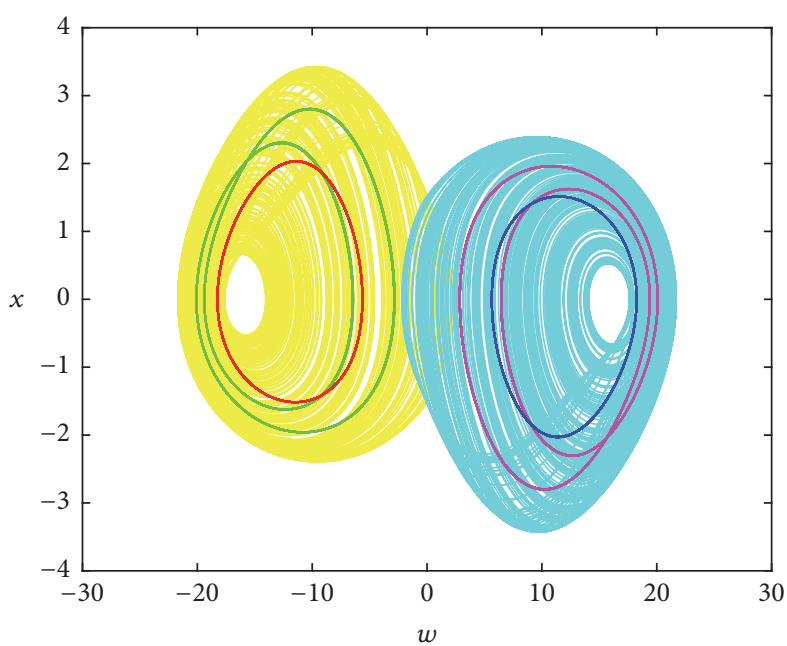

(a)

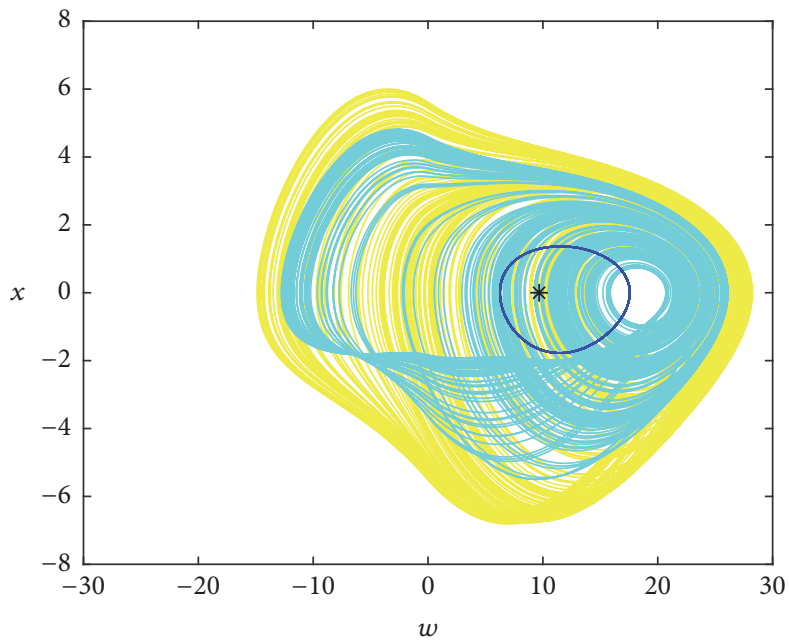

(c)

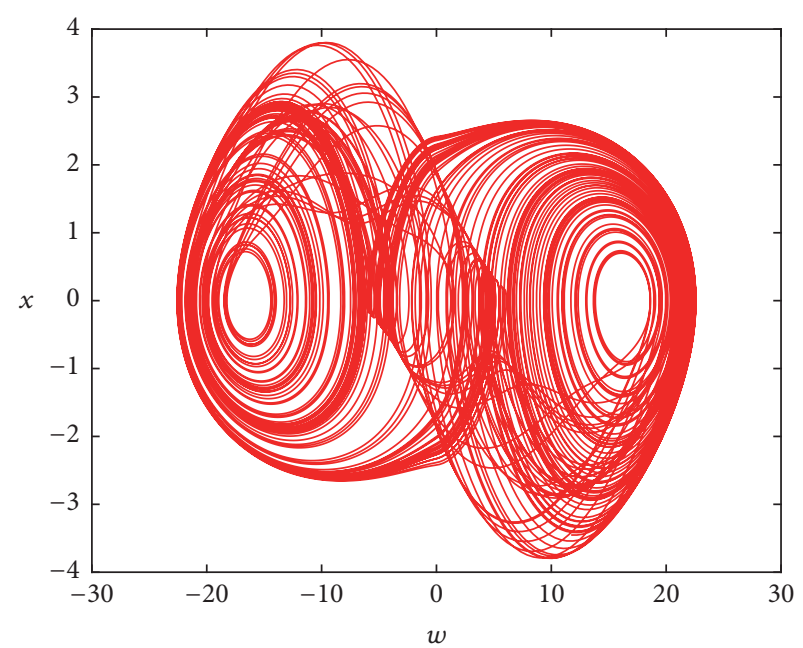

(b)

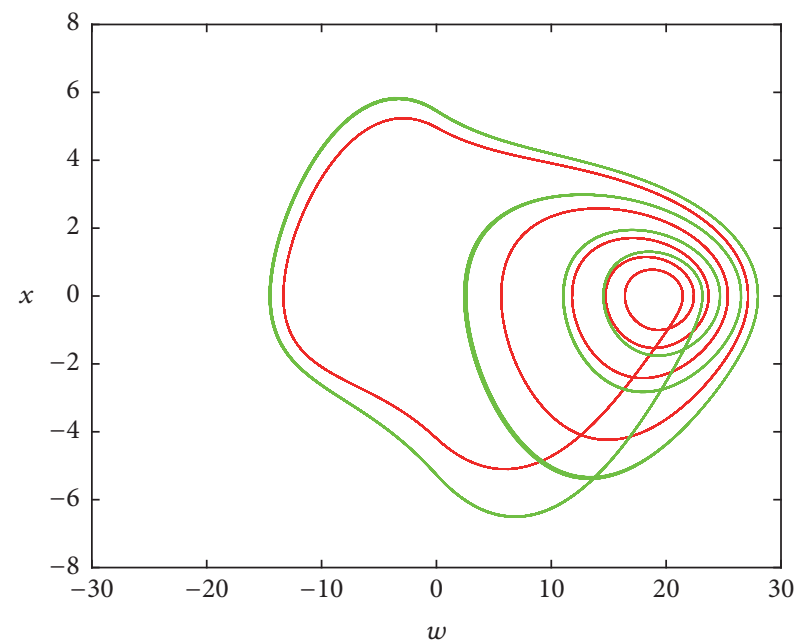

(d)

FIGURE 7: Phase portraits of various coexisting attractors in the $w$ - $x$ plane for different memristor initial conditions, where $x(0)=0, y(0)=$ 0 , and $z(0)=10^{-9}$. (a) $w(0)=-2.4$ (red) and 2.4 (blue), left-right period 1 limit cycles; $w(0)=-1.4$ (green) and 1.4 (magenta), left-right period 2 limit cycles; $w(0)=-0.5$ (yellow) and 0.5 (cyan), left-right chaotic spiral attractors; (b) $w(0)=0.001$ (red), double-scroll chaotic attractor; (c) $w(0)=-7$ (yellow), chaotic spiral attractor; $w(0)=7$ (black asterisk), point attractor; $w(0)=-2.8($ cyan), chaotic spiral attractor; $w(0)=$ 2.8 (blue), period 1 limit cycle; (d) $w(0)=-6.1$ (green), period 4 limit cycle; $w(0)=-4.3$ (red), period 5 limit cycle.

respectively. In the regions $[-2,-0.9)$ and $(0.9,2]$, several symmetrical periodic windows with different periodicities can be also seen from Figure 9. Consequently, Figure 9 shows a global symmetrical bifurcation behavior with respect to $x(0)=0$, and the phase portraits under the initial conditions of $\left(-x(0), 0,10^{-9}, 0\right)$ and $\left(x(0), 0,10^{-9}, 0\right)$ are symmetric about the origin, which confirms coexisting infinitely many attractors' behavior with the continuous variation of the initial condition $x(0)$.

When three initial conditions are chosen as $x(0)=0, z(0)$ $=0$, and $w(0)=10^{-9}$, and $y(0)$ is gradually adjusted from -1.6 to 1.6, the bifurcation diagram is shown in Figure 11(a). Similarly, when three initial conditions are set as $x(0)=$ $0, y(0)=0$, and $w(0)=10^{-9}$, and $z(0)$ is varied from -1.6 to 1.6 , the bifurcation diagram is depicted in Figure 11(b). In Figures 11(a) and 11(b), there exists symmetrical bifurcation behavior with similar dynamics including chaos, period, forward and reverse period doubling bifurcation, tangential bifurcation, chaotic crisis, and periodic windows.

4.3. Confirmation by PSIM Circuit Simulations. Considering that it is difficult to achieve the specified initial voltages of four capacitors in hardware circuit experiment, PSIM 9.0.3 is utilized to validate the coexisting attractors for some given initial voltages $[20,29,30]$.

The circuit simulation model of the memristor-based Wien-bridge oscillator is established, as shown in Figure 12. Take the results of numerical simulations in Figure 10 as examples. Corresponding to several specified initial conditions of $x(0)$, PSIM circuit simulations are performed, as plotted in Figure 13. Comparing Figure 13 


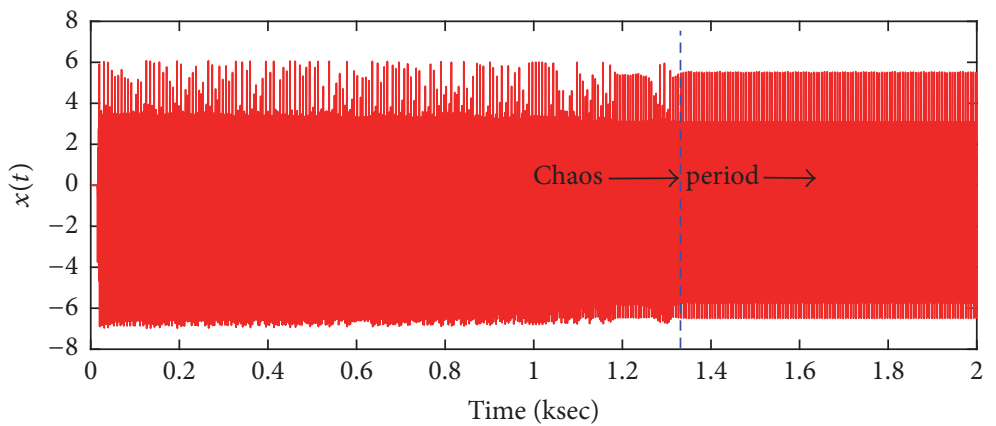

(a)

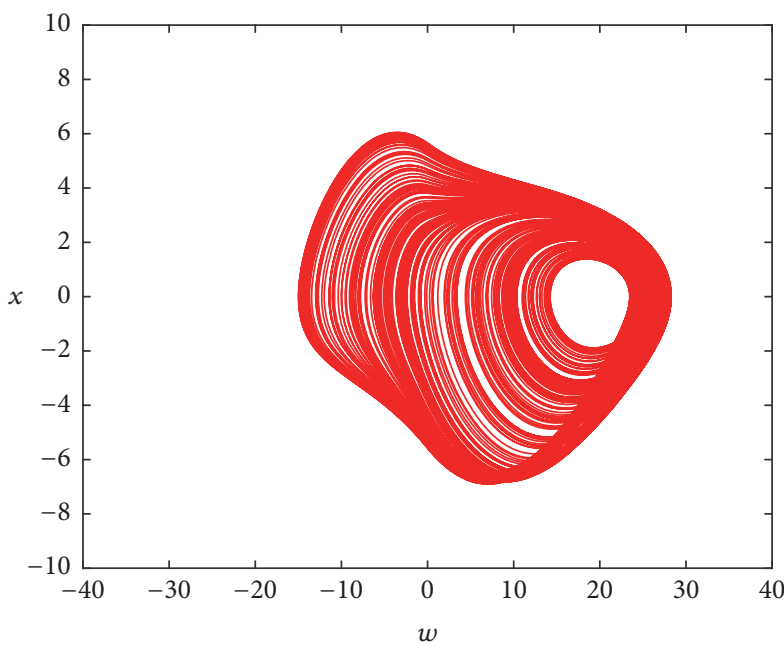

(b)

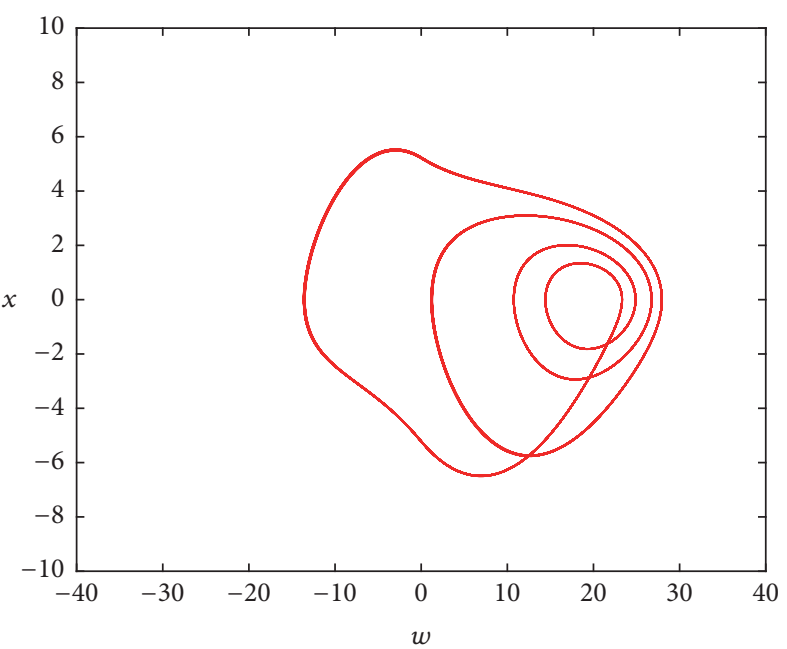

(c)

Figure 8: Numerical simulated transition behavior for $w(0)=-7.35$. (a) Time-domain waveform in the time interval [0 ks, $2 \mathrm{ks}$; (b) phase portrait of the chaotic spiral attractor in the time interval [0.6 ks, $1 \mathrm{ks}]$; (c) phase portrait of the period 4 limit cycle in the time interval [1.6 ks, $2 \mathrm{ks}]$.

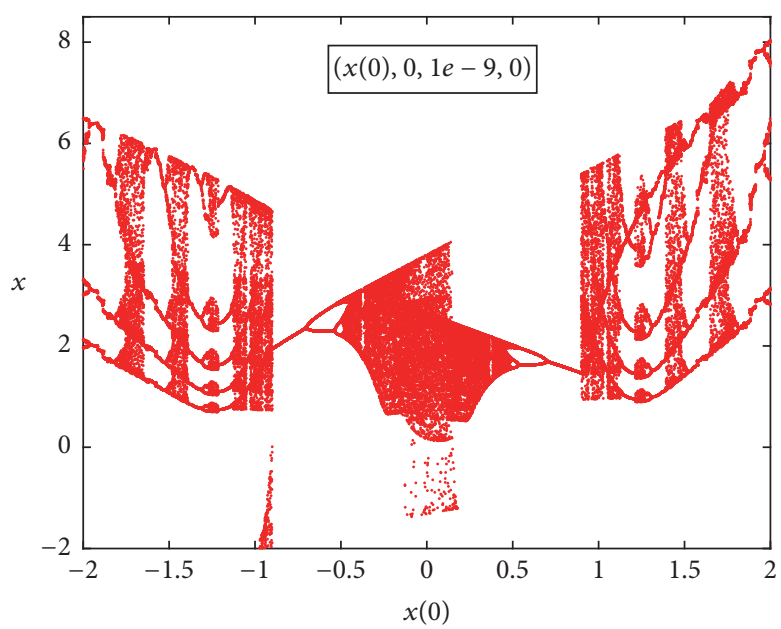

(a)

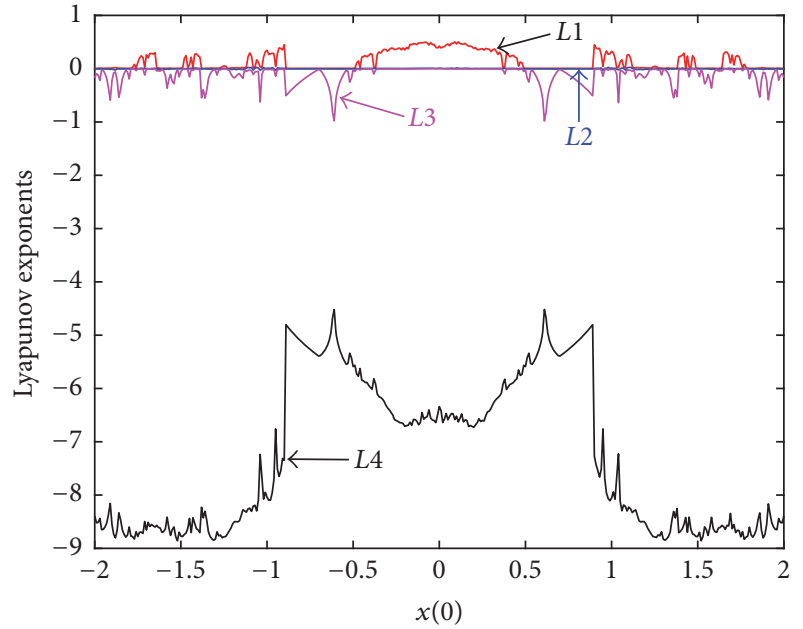

(b)

Figure 9: Dynamics with the variation of $x(0)$. (a) Bifurcation diagram; (b) Lyapunov exponent spectra. 


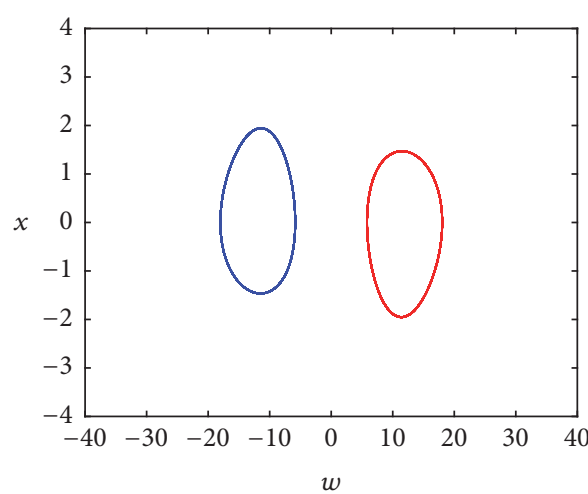

(a)

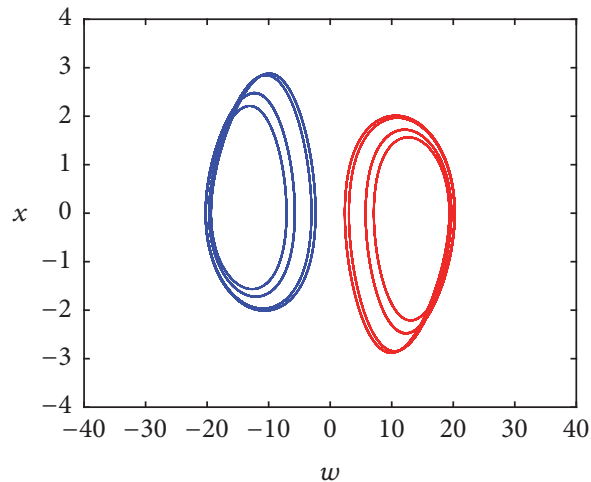

(c)

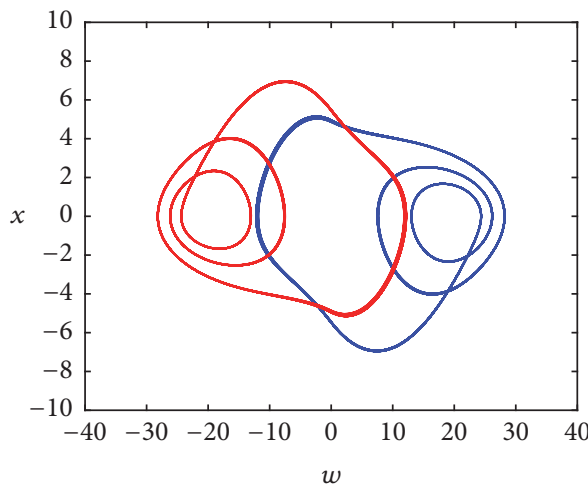

(e)

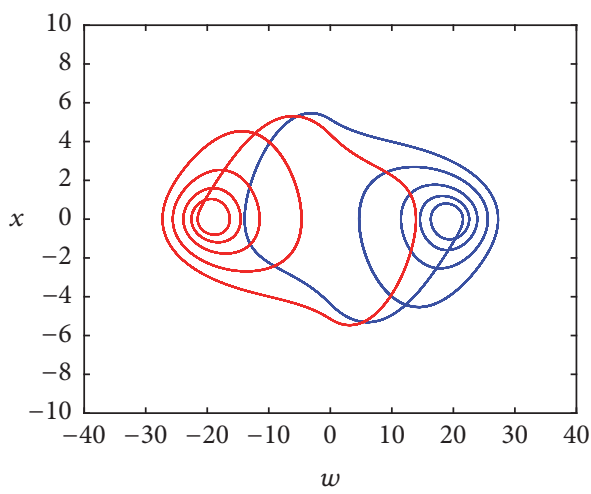

(g)

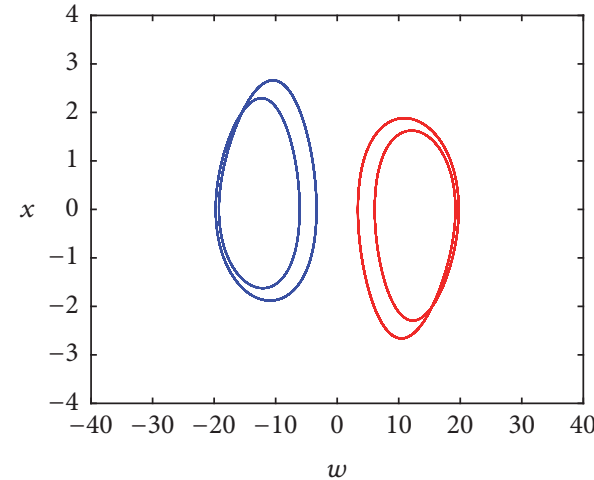

(b)

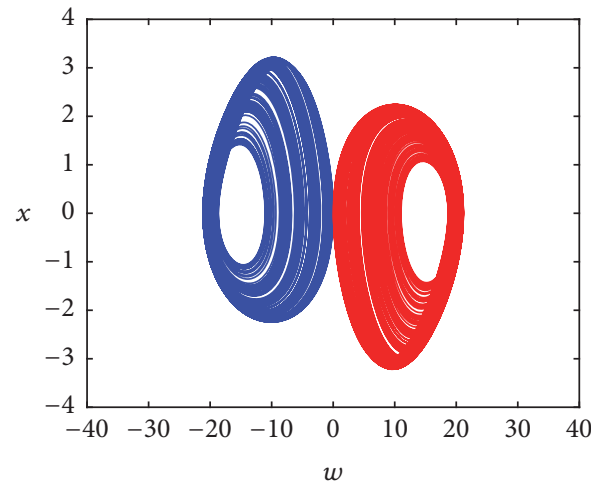

(d)

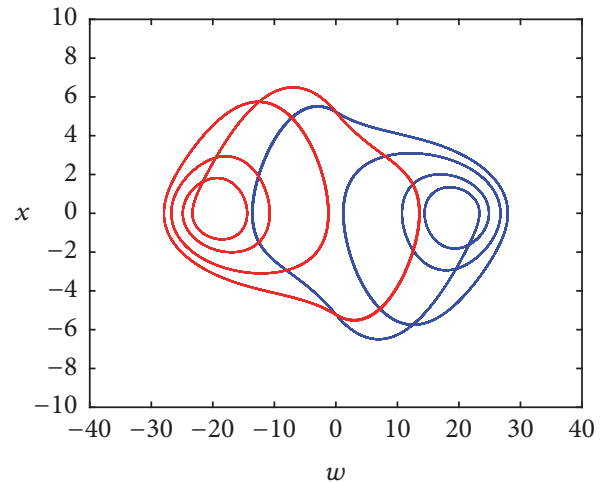

(f)

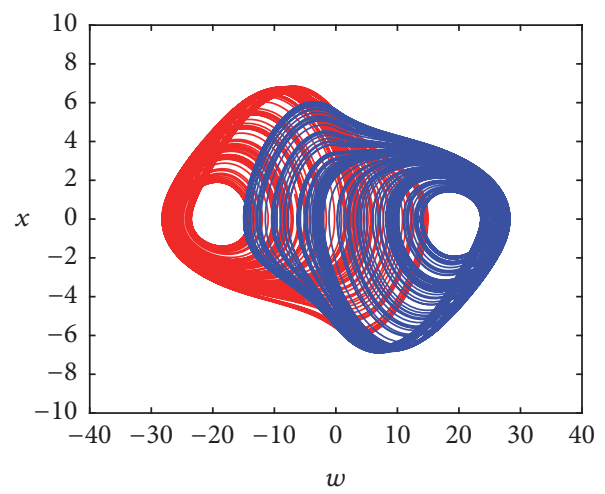

(h)

FIGURE 10: Phase portraits of various attractors in the $w$ - $x$ plane under some initial conditions $\left(x(0), 0,10^{-9}, 0\right)$. (a) Left-right period 1 limit cycles at $x(0)= \pm 0.9$; (b) left-right period 2 limit cycles at $x(0)= \pm 0.6$; (c) left-right period 4 limit cycles at $x(0)= \pm 0.5$; (d) left-right chaotic spiral attractors at $x(0)= \pm 0.32$; (e) left-right period 3 limit cycles at $x(0)= \pm 1.85$; (f) left-right period 4 limit cycles at $x(0)= \pm 1.65$; (g) left-right period 5 limit cycles at $x(0)= \pm 1.35$; (h) left-right chaotic spiral attractor at $x(0)= \pm 1.7$. 


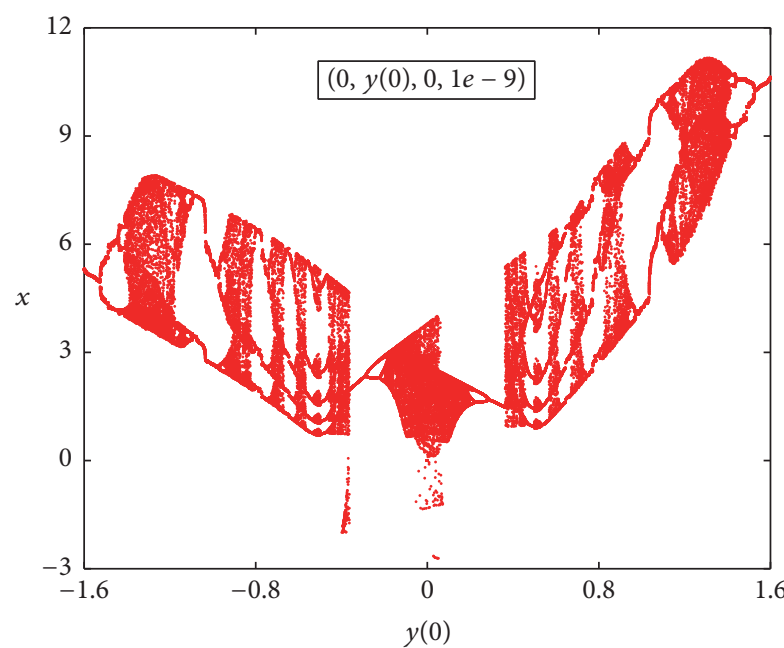

(a)

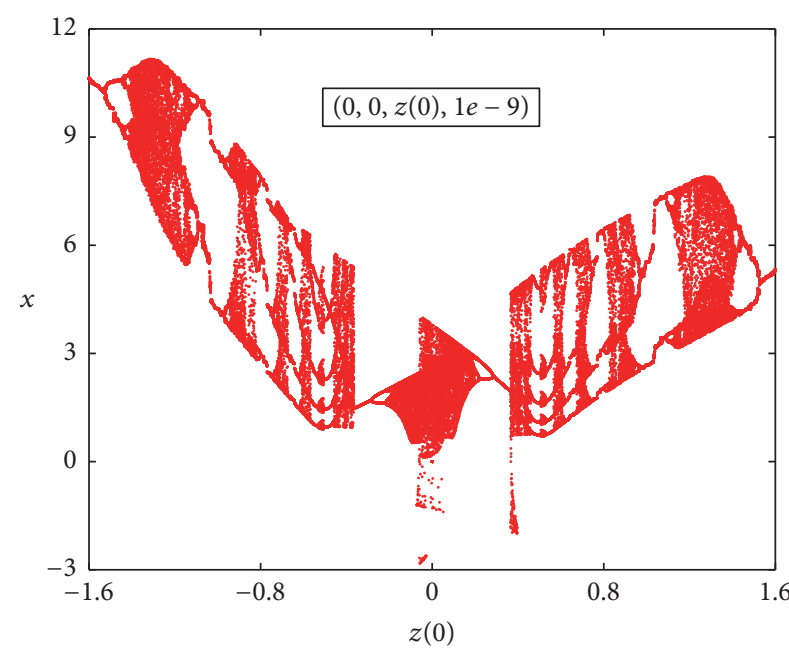

(b)

Figure 11: Bifurcation diagrams with the variations of the initial conditions $y(0)$ and $z(0)$. (a) $y(0)$ in the region $[-1.6,1.6]$; (b) $z(0)$ in the region $[-1.6,1.6]$.

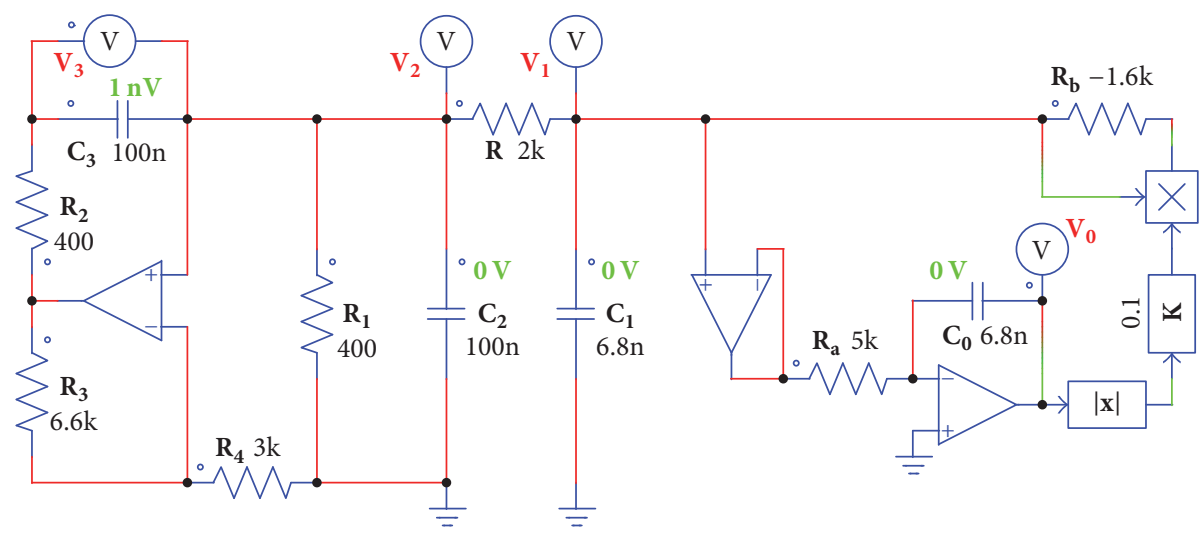

FIGURE 12: PSIM circuit simulation model, where the green texts are the four capacitor initial voltages and the red texts represent the four capacitor state voltages.

with Figure 10, it can be seen that PSIM circuit simulations are well consistent with numerical simulations, which conform that the coexisting phenomenon of infinitely many attractors indeed exists in the memristor-based Wien-bridge oscillator.

\section{Conclusions}

When a nonlinear active filter consisting of an active voltagecontrolled memristor and a capacitor is connected to a classical Wien-bridge oscillator in parallel, a memristorbased Wien-bridge chaotic oscillator is constructed. The proposed memristive oscillator has a line equilibrium and thereby its dynamical behaviors depend on the circuit parameters and the initial conditions. Focusing on the parameterindependent dynamical behaviors in the memristive oscillator, a set of circuit parameters are determined and only the initial condition-dependent dynamical behaviors are explored by using some conventional methods of bifurcation diagrams, Lyapunov exponents, and phase portraits, from which the extreme multistability phenomenon of coexisting infinitely many attractors is effectively exhibited. Furthermore, hardware experiments and PSIM circuit simulations are performed to well verify the numerical simulations. Such a work may be attributed to some technological applications, for an example, multirandom signal generator, in future days.

\section{Conflicts of Interest}

The authors declare that there are no conflicts of interest regarding the publication of this paper.

\section{Acknowledgments}

This work was supported by the National Natural Science Foundations of China under Grant nos. 51777016, 61601062, 


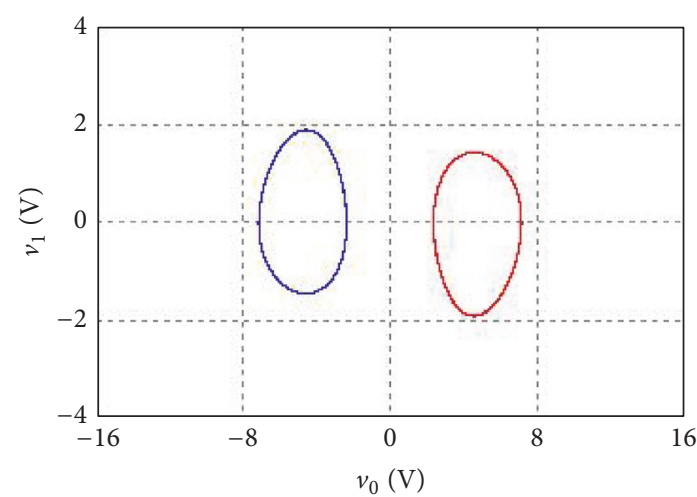

(a)

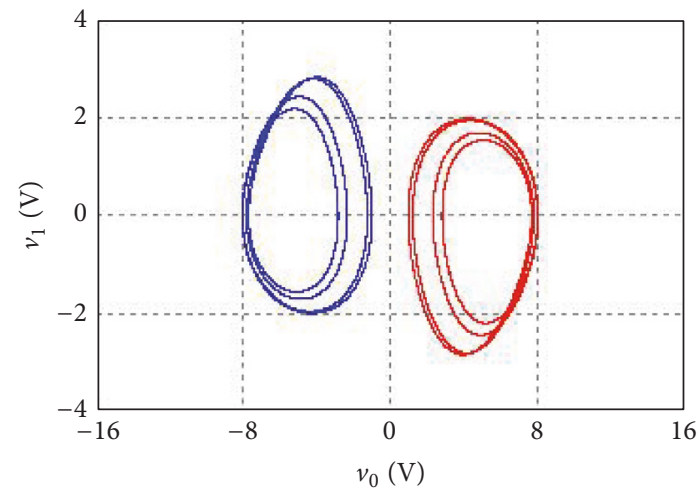

(c)

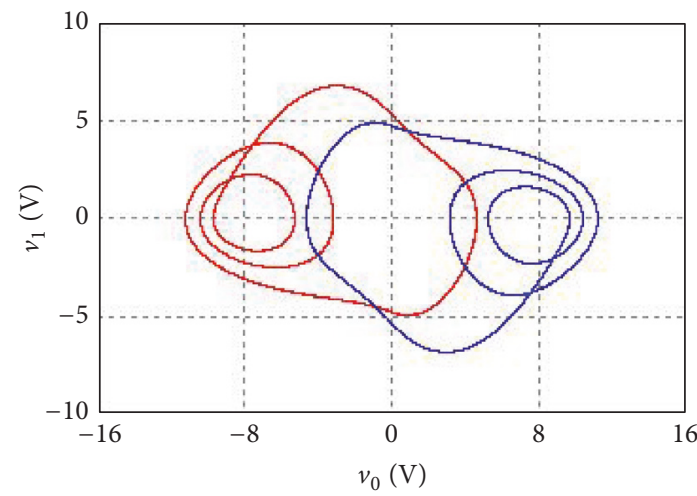

(e)

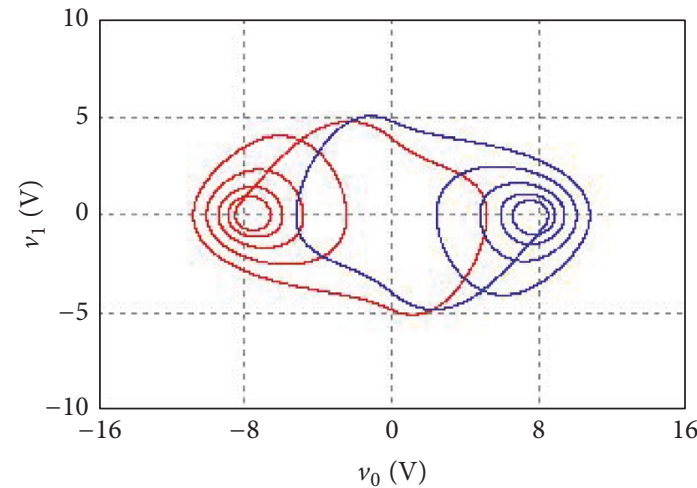

(g)

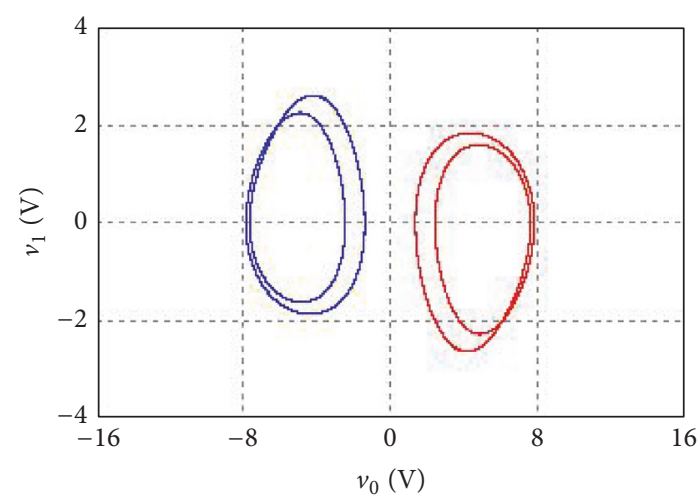

(b)

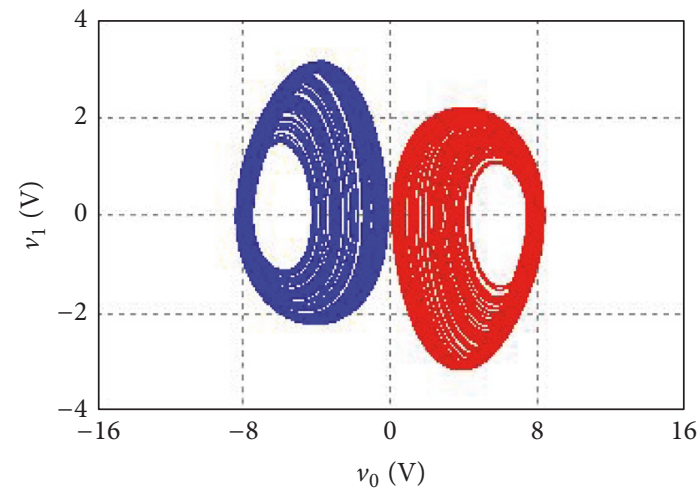

(d)

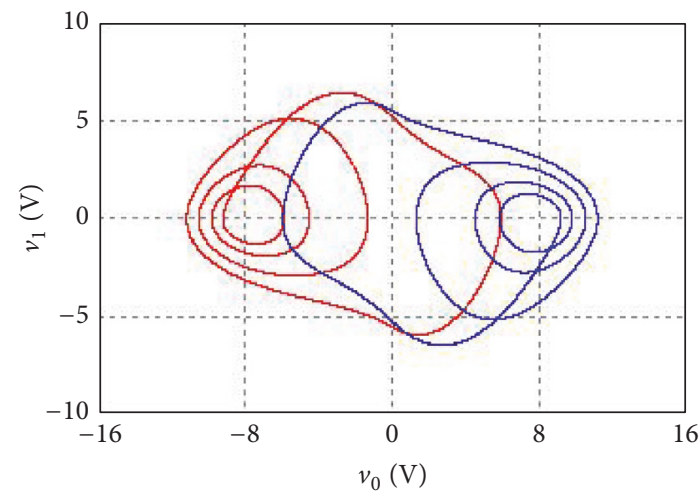

(f)

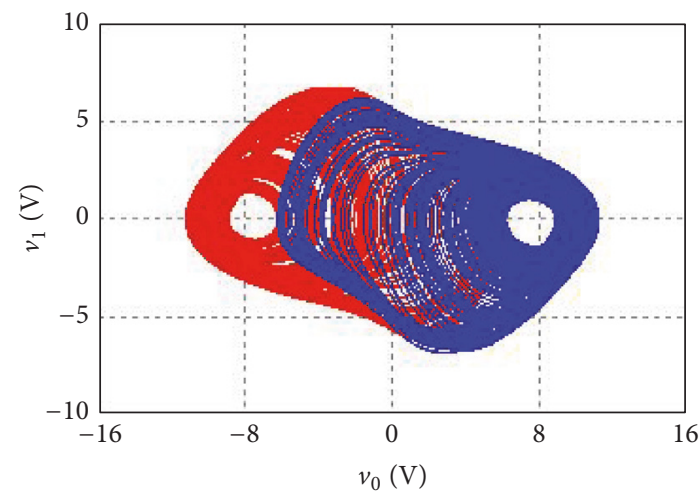

(h)

FIGURE 13: Phase portraits of various attractors in the $v_{0}-v_{1}$ plane under several specified initial conditions of $\left(v_{1}(0) \mathrm{V}, 0 \mathrm{~V}, 10^{-9} \mathrm{~V}, 0 \mathrm{~V}\right)$. (a) Left-right period-1 limit cycles at $v_{1}(0)= \pm 0.9 \mathrm{~V}$; (b) left-right period 2 limit cycles at $v_{1}(0)= \pm 0.6 \mathrm{~V}$; (c) left-right period 4 limit cycles at $v_{1}(0)$ $= \pm 0.5 \mathrm{~V}$; (d) left-right chaotic spiral attractors at $v_{1}(0)= \pm 0.32 \mathrm{~V}$; (e) left-right period 3 limit cycles at $v_{1}(0)= \pm 1.85 \mathrm{~V}$; (f) left-right period 4 limit cycles at $v_{1}(0)= \pm 1.65 \mathrm{~V}$; (g) left-right period 5 limit cycles at $v_{1}(0)= \pm 1.35 \mathrm{~V}$; (h) left-right chaotic spiral attractor at $v_{1}(0)= \pm 1.7 \mathrm{~V}$. 
and 51607013 and the Natural Science Foundation of Jiangsu Province, China, under Grant no. BK20160282.

\section{References}

[1] L. O. Chua, "Memristor-the missing circuit element," IEEE Transactions on Circuit Theory, vol. 18, no. 5, pp. 507-519, 1971.

[2] B.-C. Bao, J.-P. Xu, G.-H. Zhou, Z.-H. Ma, and L. Zou, "Chaotic memristive circuit: equivalent circuit realization and dynamical analysis," Chinese Physics B, vol. 20, Article ID 120502, 2011.

[3] B.-C. Bao, F.-W. Hu, Z. Liu, and J.-P. Xu, "Mapping equivalent approach to analysis and realization of memristor-based dynamical circuit," Chinese Physics B, vol. 23, no. 7, Article ID 070503, 2014.

[4] A. Buscarino, L. Fortuna, M. Frasca, and L. Valentina Gambuzza, "A chaotic circuit based on Hewlett-Packard memristor," Chaos: An Interdisciplinary Journal of Nonlinear Science, vol. 22, no. 2, Article ID 023136, 2012.

[5] G.-Y. Wang, J.-L. He, F. Yuan, and C.-J. Peng, "Dynamical behaviors of a $\mathrm{TiO}_{2}$ memristor oscillator," Chinese Physics Letters, vol. 30, no. 11, 2013.

[6] Z.-J. Li and Y.-C. Zeng, "A memristor oscillator based on a twinT network," Chinese Physics B, vol. 22, no. 4, Article ID 040502, 2013.

[7] A. L. Fitch, D. Yu, H. H. C. Iu, and V. Sreeram, "Hyperchaos in a memristor-based modified canonical Chua's circuit," International Journal of Bifurcation and Chaos, vol. 22, no. 6, Article ID 1250133, 2012.

[8] B. Bao, P. Jiang, H. Wu, and F. Hu, "Complex transient dynamics in periodically forced memristive Chua's circuit," Nonlinear Dynamics, vol. 79, no. 4, pp. 2333-2343, 2015.

[9] M. Chen, M. Li, Q. Yu, B. Bao, Q. Xu, and J. Wang, "Dynamics of self-excited attractors and hidden attractors in generalized memristor-based Chua's circuit," Nonlinear Dynamics, vol. 81, no. 1-2, pp. 215-226, 2015.

[10] M. Chen, J. Yu, and B.-C. Bao, "Finding hidden attractors in improved memristor-based Chua's circuit," IEEE Electronics Letters, vol. 51, no. 6, pp. 462-464, 2015.

[11] V. Venkatasubramanian, "Coexistence of four different attractors in a fundamental power system model," IEEE Transactions on Circuits and Systems I: Fundamental Theory and Applications, vol. 46, no. 3, pp. 405-409, 1999.

[12] J. Argyris and I. Andreadis, "On the influence of noise on the coexistence of chaotic attractors," Chaos, Solitons \& Fractals, vol. 11, no. 6, pp. 941-946, 2000.

[13] S. Arathi and S. Rajasekar, "Stochastic resonance in a single-well anharmonic oscillator with coexisting attractors," Communications in Nonlinear Science and Numerical Simulation, vol. 19, no. 12, pp. 4049-4056, 2014.

[14] J. C. Sprott, X. Wang, and G. Chen, "Coexistence of point, periodic and strange attractors," International Journal of Bifurcation and Chaos, vol. 23, no. 5, Article ID 1350093, 2013.

[15] C. Li and J. C. Sprott, "Multistability in the lorenz system: a broken butterfly," International Journal of Bifurcation and Chaos, vol. 24, no. 10, Article ID 1450131, 2014.

[16] J. Kengne, Z. N. Tabekoueng, V. K. Tamba, and A. N. Negou, "Periodicity, chaos, and multiple attractors in a memristorbased Shinriki's circuit," Chaos: An Interdisciplinary Journal of Nonlinear Science, vol. 25, no. 10, Article ID 103126, 2015.

[17] Q. Xu, Y. Lin, B. Bao, and M. Chen, "Multiple attractors in a nonideal active voltage-controlled memristor based Chua's circuit," Chaos, Solitons \& Fractals, vol. 83, pp. 186-200, 2016.
[18] Z. T. Njitacke, J. Kengne, and A. Nguomkam Negou, "Dynamical analysis and electronic circuit realization of an equilibrium free 3D chaotic system with a large number of coexisting attractors," Optik-International Journal for Light and Electron Optics, vol. 130, pp. 356-364, 2017.

[19] B. C. Bao, Q. D. Li, N. Wang, and Q. Xu, "Multistability in Chua's circuit with two stable node-foci," Chaos: An Interdisciplinary Journal of Nonlinear Science, vol. 26, no. 4, Article ID 043111, 2016.

[20] J. Ma, F. Wu, G. Ren, and J. Tang, "A class of initials-dependent dynamical systems," Applied Mathematics and Computation, vol. 298, pp. 65-76, 2017.

[21] Q. Xu, Q. Zhang, N. Wang, H. Wu, and B. Bao, "An improved memristive diode bridge-based band pass filter chaotic circuit," Mathematical Problems in Engineering, vol. 2017, Article ID 2461964, 11 pages, 2017.

[22] Z. Li, M. Ma, M. Wang, and Y. Zeng, "Realization of currentmode SC-CNN-based Chua's circuit," AEU-International Journal of Electronics and Communications, vol. 71, pp. 21-29, 2017.

[23] M. Di Marco, M. Forti, and L. Pancioni, "Convergence and multistability of nonsymmetric cellular neural networks with memristors," IEEE Transactions on Cybernetics, vol. 47, no. 10, pp. 2970-2983, 2017.

[24] B. Bao, H. Qian, J. Wang et al., "Numerical analyses and experimental validations of coexisting multiple attractors in Hopfield neural network," Nonlinear Dynamics, vol. 90, no. 4, pp. 2359-2369, 2017.

[25] B. Bao, H. Qian, Q. Xu, M. Chen, J. Wang, and Y. Yu, "Coexisting behaviors of asymmetric attractors in hyperbolictype memristor based hopfield neural network," Frontiers in Computational Neuroscience, vol. 11, no. 81, pp. 1-14, 2017.

[26] M. S. Patel, U. Patel, A. Sen et al., "Experimental observation of extreme multistability in an electronic system of two coupled Rössler oscillators," Physical Review E: Statistical, Nonlinear, and Soft Matter Physics, vol. 89, no. 2, Article ID 022918, 2014.

[27] F. Yuan, G. Wang, and X. Wang, "Extreme multistability in a memristor-based multi-scroll hyper-chaotic system," Chaos: An Interdisciplinary Journal of Nonlinear Science, vol. 26, no. 7, Article ID 073107, 2016.

[28] G. Wang, C. Shi, X. Wang, and F. Yuan, "Coexisting oscillation and extreme multistability for a memcapacitor-based circuit," Mathematical Problems in Engineering, vol. 2017, Article ID 6504969, 13 pages, 2017.

[29] B. Bao, T. Jiang, Q. Xu, M. Chen, H. Wu, and Y. Hu, "Coexisting infinitely many attractors in active band-pass filter-based memristive circuit," Nonlinear Dynamics, vol. 86, no. 3, pp. 1711-1723, 2016.

[30] B. C. Bao, H. Bao, N. Wang, M. Chen, and Q. Xu, "Hidden extreme multistability in memristive hyperchaotic system," Chaos, Solitons \& Fractals, vol. 94, pp. 102-111, 2017.

[31] B. Bao, T. Jiang, G. Wang, P. Jin, H. Bao, and M. Chen, “Twomemristor-based Chua's hyperchaotic circuit with plane equilibrium and its extreme multistability," Nonlinear Dynamics, vol. 89, no. 2, pp. 1157-1171, 2017.

[32] F. Corinto and M. Forti, "Memristor circuits: flux-charge analysis method," IEEE Transactions on Circuits and Systems I: Regular Papers, vol. 63, no. 11, pp. 1997-2009, 2016.

[33] C. Fernando and F. Mauro, "Memristor circuits: bifurcations without parameters," IEEE Transactions on Circuits and Systems I: Regular Papers, vol. 64, no. 6, pp. 1540-1551, 2016. 
[34] M. Chen, M. Sun, B. Bao, H. Wu, Q. Xu, and J. Wang, "Controlling extreme multistability of memristor emulatorbased dynamical circuit in flux-charge domain," Nonlinear Dynamics, 2017.

[35] V. N. Chizhevsky, "Coexisting attractors in a $\mathrm{CO}_{2}$ laser with modulated losses," Journal of Optics B: Quantum and Semiclassical Optics, vol. 2, no. 6, pp. 711-717, 2000.

[36] S. Morfu, B. Nofiele, and P. Marquié, "On the use of multistability for image processing," Physics Letters A, vol. 367, no. 3, pp. 192-198, 2007.

[37] C. N. Ngonghala, U. Feudel, and K. Showalter, "Extreme multistability in a chemical model system," Physical Review E: Statistical, Nonlinear, and Soft Matter Physics, vol. 83, no. 5, Article ID 056206, 2011.

[38] M. Rabinovich, R. Huerta, and G. Laurent, "Transient dynamics for neural processing," Science, vol. 321, no. 5885, pp. 48-50, 2008.

[39] F. Romeo, L. I. Manevitch, L. A. Bergman, and A. Vakakis, "Transient and chaotic low-energy transfers in a system with bistable nonlinearity," Chaos: An Interdisciplinary Journal of Nonlinear Science, vol. 25, no. 5, Article ID 053109, 2015.

[40] X.-Y. Dang, C.-B. Li, B.-C. Bao, and H.-G. Wu, "Complex transient dynamics of hidden attractors in a simple 4D system," Chinese Physics B, vol. 24, no. 5, 2015.

[41] T. Tél, “The joy of transient chaos," Chaos: An Interdisciplinary Journal of Nonlinear Science, vol. 25, no. 9, Article ID 097619, 2015.

[42] M.-F. Danca, "Hidden transient chaotic attractors of Rabinovich-Fabrikant system," Nonlinear Dynamics, vol. 86, no. 2, pp. 1263-1270, 2016.

[43] M. Dhamala and Y.-C. Lai, "Controlling transient chaos in deterministic flows with applications to electrical power systems and ecology," Physical Review E: Statistical Physics, Plasmas, Fluids, and Related Interdisciplinary Topics, vol. 59, no. 2, pp. 1646-1655, 1999.

[44] L. O. Chua, "The double scroll family," IEEE Transactions on Circuits and Systems II: Express Briefs, vol. 33, no. 11, pp. 10721118, 1986.

[45] B. Bao, N. Wang, M. Chen, Q. Xu, and J. Wang, "Inductorfree simplified Chua's circuit only using two-op-amp-based realization," Nonlinear Dynamics, vol. 84, no. 2, pp. 511-525, 2016.

[46] A. Wolf, J. B. Swift, H. L. Swinney, and J. A. Vastano, "Determining Lyapunov exponents from a time series," Physica D: Nonlinear Phenomena, vol. 16, no. 3, pp. 285-317, 1985. 


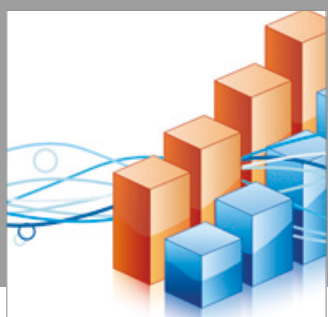

Advances in

Operations Research

vatersals

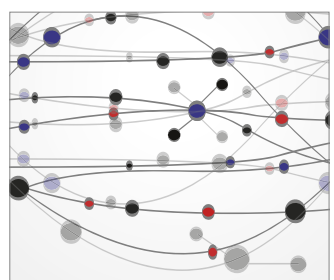

\section{The Scientific} World Journal
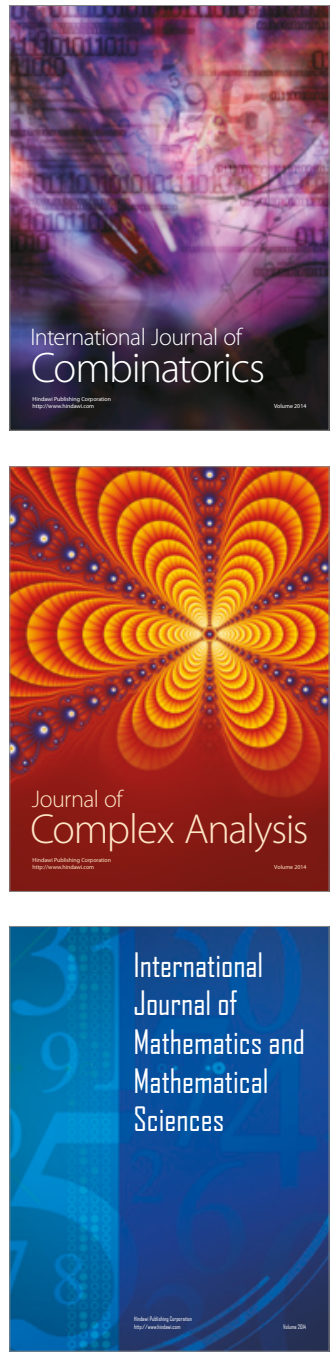
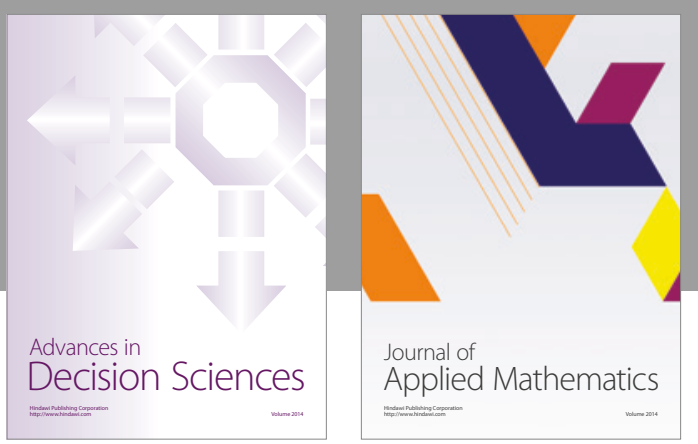

Algebra

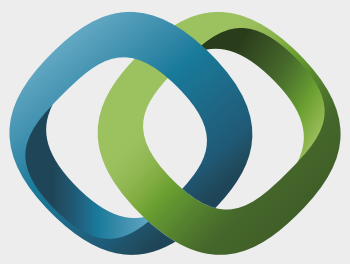

\section{Hindawi}

Submit your manuscripts at

https://www.hindawi.com
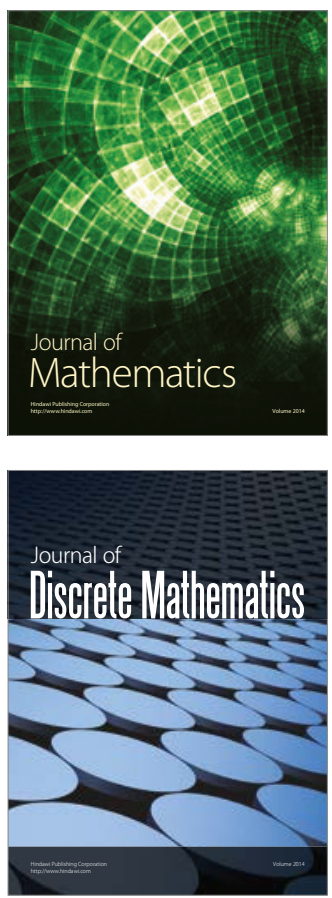

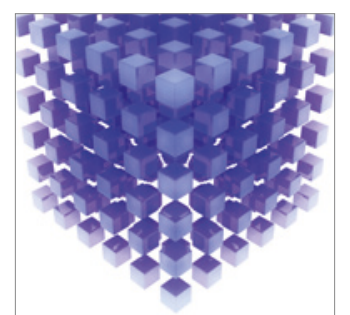

Mathematical Problems in Engineering
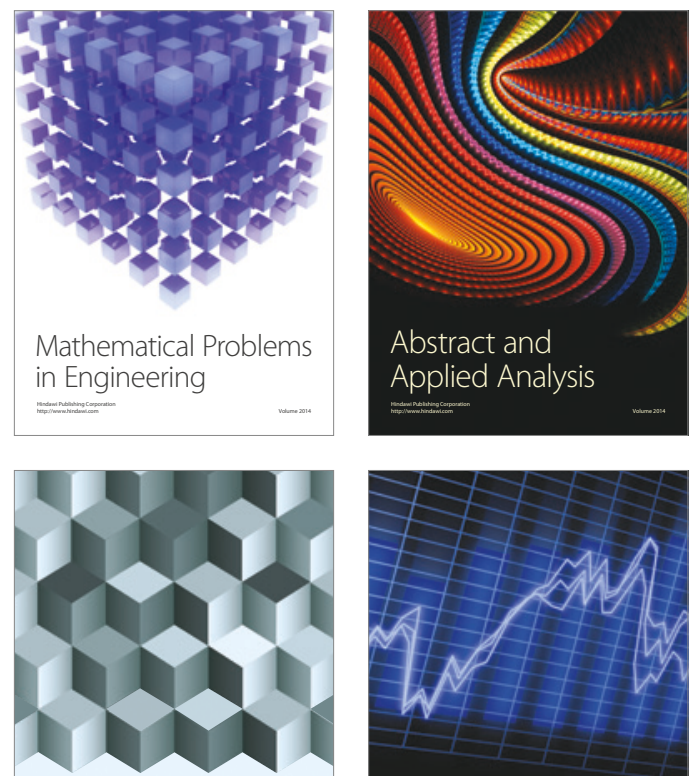

Journal of

Function Spaces

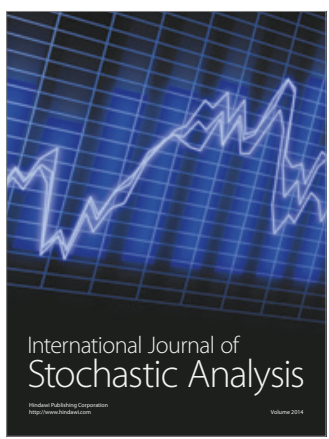

Probability and Statistics
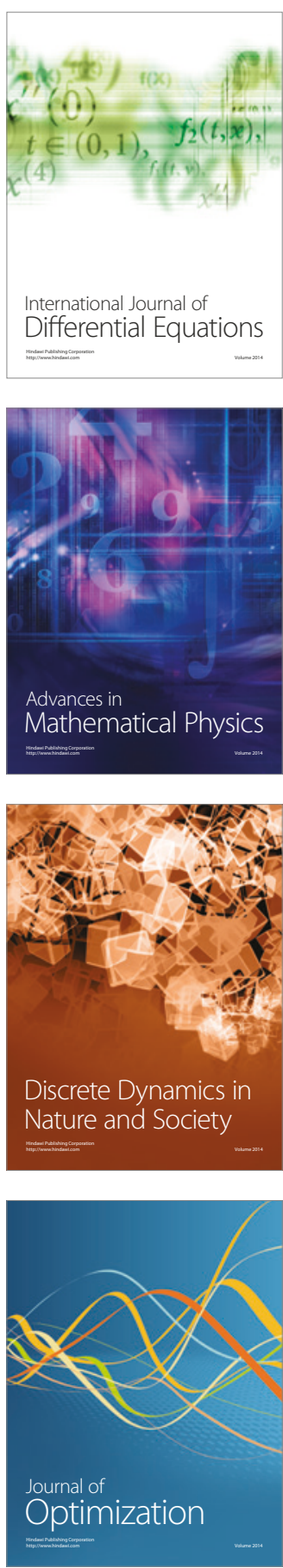